\title{
On Nonuniform Polynomial Trichotomy of Linear Discrete-Time Systems in Banach Spaces
}

\author{
Zhi-gang Li, Xiao-qiu Song, and Xiu-li Yang \\ College of Science, China University of Mining and Technology, Xuzhou, Jiangsu 221008, China \\ Correspondence should be addressed to Xiao-qiu Song; sxqcumt@163.com
}

Received 31 March 2014; Accepted 23 June 2014; Published 14 July 2014

Academic Editor: Olivier Bahn

Copyright (C) 2014 Zhi-gang Li et al. This is an open access article distributed under the Creative Commons Attribution License, which permits unrestricted use, distribution, and reproduction in any medium, provided the original work is properly cited.

\begin{abstract}
We study two nonuniform polynomial trichotomy concepts for linear discrete-time systems in Banach spaces. Our main objective is to give summation property for nonuniform polynomial trichotomies. As for applications we obtain characterization of these concepts in terms of Lyapunov functions.
\end{abstract}

\section{Introduction}

In the mathematical literature of the last decades, the asymptotic behaviors of solutions of evolution equations in finite and infinite dimensional spaces have proved to be a research area of large intensity. A number of long standing open problems have recently been solved and the theory seems to have obtained a certain degree of maturity. Some concepts of the asymptotic behaviors were defined and developed, such as stability, expansivity, dichotomy, and trichotomy (see [1-14] and the references therein), based on the fact that the dynamical systems which describe processes from economics, physical sciences, or engineering are extremely complex and the identification of the proper mathematical model is difficult.

The conception of trichotomy firstly arose in the works of Sacker and Sell [7] in 1976. They described trichotomy for linear differential systems by linear skew-product flows. Later, Elaydi and Hájek [11, 12] gave the notions of exponential trichotomy for differential systems and for nonlinear differential systems, respectively. The case of difference equations received special attention in the paper of Elaydi and Janglajew [13] where the authors deduced the first input-output criteria for exponential trichotomy, and they introduced the first nonlinear discrete concepts of exponential trichotomy.

As a natural generalization of exponential dichotomy (firstly introduced by Perron in [6]), exponential trichotomy (see $[4,9,14]$ and the references therein) is one of the most complex asymptotic properties of dynamical systems arising from the central manifold theory. Exponential trichotomy is a powerful tool for analyzing the asymptotic behavior of dynamical systems. In the study of the trichotomy, the main idea is to obtain a decomposition of the space at every moment into three closed subspaces: the stable subspace, the unstable subspace, and the center manifold (see [8]). Thus, with motivation from the properties arising in bifurcation theory, a new asymptotic concept called exponential trichotomy which reflects a deeper analysis of the behavior of solutions of dynamical systems is introduced, and Barreira and Valls found that each nonautonomous system could generalize a family of Lyapunov functions (see [10]).

The existence of exponential trichotomies is a strong requirement and hence it is of considerable interest to look for more general types of trichotomic behaviors. Recently, a notion of nonuniform polynomial dichotomy was introduced independently by Barreira and Valls in [1] and Bento and Silva in [2] in somewhat distinct forms, respectively, in the case of continuous and discrete-time systems. Ramneanţu et al. had offered some integral properties for nonuniform polynomial dichotomy in [3]. In this case the rates of contraction and expansion vary polynomially. The same as dichotomy, we would like to obtain some properties of polynomial trichotomy. In this paper we investigate two nonuniform polynomial trichotomy concepts for the general case of linear 
discrete-time system in Banach spaces. The case of uniform polynomial trichotomy has been discussed by Ramneanţu in [5]. Our approach is based on the extension of exponential trichotomy to the case of polynomial trichotomy.

\section{Preliminaries}

Let $X$ be a real or complex Banach space. The norm on $X$ and on $\mathscr{B}(X)$, the Banach algebra of all bounded linear operators acting on $X$, will be denoted by $\|\cdot\|$. We denote $\Delta=\{(m, n) \in$ $\left.\mathbb{N}^{2}, m \geq n\right\}$ and $T=\left\{(m, r, n) \in \mathbb{N}^{3}, m \geq r \geq n\right\}$. Let $\mathscr{F}$ be the set of all nondecreasing functions $F: \mathbb{R}_{+} \rightarrow \mathbb{R}_{+}$with the properties $F(0)=0$ and $F(t)>0$ for every $t>0$. Let $I$ be the identity operator on $X$.

In the present paper we consider linear discrete-time system of difference equations

$$
x_{n+1}=A(n) x_{n}, \quad n \in \mathbb{N},
$$

where $A: \mathbb{N} \rightarrow \mathscr{B}(X)$ is a sequence in $\mathscr{B}(X)$. Then every solution $x=\left\{x_{n}\right\}$ of the system (1) is given by

$$
x_{m}=U(m, n) x_{n}
$$

for all $(m, n) \in \Delta$, where the mapping $U: \Delta \rightarrow \mathscr{B}(X)$ is defined by

$$
U(m, n):= \begin{cases}I, & m=n \\ A(m-1) \cdots A(n), & m>n .\end{cases}
$$

It is easy to see that $U(m, r) U(r, n)=U(m, n)$, for all $(m, r, n)$ $\in T$.

For the particular case when (1) is autonomous, that is, $A(n)=A \in \mathscr{B}(X)$ for all $n \in \mathbb{N}$, then $U(m, n)=A^{m-n}$ for all $(m, n) \in \Delta$.

Definition 1. An application $P: \mathbb{N} \rightarrow \mathscr{B}(X)$ is said to be a projection family on $X$ if

$$
P^{2}(n)=P(n),
$$

for all $n \in \mathbb{N}$.

Definition 2. Three projection families $P_{1}, P_{2}, P_{3}: \mathbb{N} \rightarrow$ $\mathscr{B}(X)$ are said to be compatible with the system (1), if

(c1) $P_{1}(n)+P_{2}(n)+P_{3}(n)=I$, for all $n \in \mathbb{N}$;

(c2) $P_{i}(n) P_{j}(n)=0$, for all $n \in \mathbb{N}$, for all $i, j \in\{1,2,3\}$, $i \neq j$

(c3) $A(n) P_{i}(n)=P_{i}(n+1) A(n)$, for all $n \in \mathbb{N}$ and all $i \in$ $\{1,2,3\}$.

Remark 3. If the projection families $P_{1}, P_{2}, P_{3}: \mathbb{N} \rightarrow \mathscr{B}(X)$ are compatible with the system (1) then we have

$$
U(m, n) P_{i}(n)=P_{i}(m) U(m, n)
$$

for all $n \in \mathbb{N}, i \in\{1,2,3\}$.

We denote that system (1) is nonuniform trichotomy if there exist a nondecreasing sequence of real numbers $N$ : $\mathbb{N} \rightarrow \mathbb{R}_{+}^{*}$, such that

$$
\begin{aligned}
& \left(\mathrm{nt}_{1}\right) \quad\left\|U(m, n) P_{1}(n) x\right\| \leq N(n)\left\|P_{1}(n) x\right\|, \\
& \left(\mathrm{nt}_{2}\right) \quad\left\|P_{2}(n) x\right\| \leq N(m)\left\|U(m, n) P_{2}(n) x\right\|, \\
& \left(\mathrm{nt}_{3}\right)\left\|U(m, n) P_{3}(n) x\right\| \leq N(n)\left\|P_{3}(n) x\right\|, \\
& \left(\mathrm{nt}_{4}\right)\left\|P_{3}(n) x\right\| \leq N(m)\left\|U(m, n) P_{3}(n) x\right\|,
\end{aligned}
$$

for all $(m, n, x) \in \Delta \times X$.

\section{Exponential Trichotomy Concepts}

Definition 4. The linear discrete-time system (1) is said to be uniformly exponentially trichotomic if there exist three projection families $\left\{P_{i}\right\}_{i \in\{1,2,3\}}$ compatible with the system (1), constants $N \geq 1$, and $v_{1}, v_{2}, v_{3}$, and $v_{4}$ with the property $v_{1} \leq v_{2}<0<v_{3} \leq v_{4}$ such that

$$
\begin{array}{ll}
\left(\text { uet }_{1}\right) & \left\|U(m, n) P_{1}(n) x\right\| \leq N e^{v_{1}(m-n)}\left\|P_{1}(n) x\right\|, \\
\left(\text { uet }_{2}\right) & \left\|P_{2}(n) x\right\| \leq N e^{-v_{4}(m-n)}\left\|U(m, n) P_{2}(n) x\right\|, \\
\text { (uet } \left._{3}\right) & \left\|U(m, n) P_{3}(n) x\right\| \leq N e^{v_{3}(m-n)}\left\|P_{3}(n) x\right\|, \\
\text { uet } \left._{4}\right) & \left\|P_{3}(n) x\right\| \leq N e^{-v_{2}(m-n)}\left\|U(m, n) P_{3}(n) x\right\|,
\end{array}
$$

for all $(m, n, x) \in \Delta \times X$.

Definition 5. The linear discrete-time system (1) is said to be nonuniformly exponentially trichotomic if there exist three projection families $\left\{P_{i}\right\}_{i \in\{1,2,3\}}$ compatible with the system (1), a nondecreasing sequence of real numbers $N: \mathbb{N} \rightarrow \mathbb{R}_{+}^{*}$, and constants $v_{1}, v_{2}, v_{3}$, and $v_{4}$ with the property $v_{1} \leq v_{2}<$ $0<v_{3} \leq v_{4}$ such that

$$
\begin{aligned}
& \text { (net } \left.{ }_{1}\right)\left\|U(m, n) P_{1}(n) x\right\| \leq N(n) e^{v_{1}(m-n)}\left\|P_{1}(n) x\right\| \text {, } \\
& \left(\text { net }_{2}\right)\left\|P_{2}(n) x\right\| \leq N(m) e^{-v_{4}(m-n)}\left\|U(m, n) P_{2}(n) x\right\| \text {, } \\
& \left(\text { net }_{3}\right)\left\|U(m, n) P_{3}(n) x\right\| \leq N(n) e^{v_{3}(m-n)}\left\|P_{3}(n) x\right\| \text {, } \\
& \left(\text { net }_{4}\right)\left\|P_{3}(n) x\right\| \leq N(m) e^{-v_{2}(m-n)}\left\|U(m, n) P_{3}(n) x\right\| \text {, }
\end{aligned}
$$

for all $(m, n, x) \in \Delta \times X$.

Remark 6. It is obvious that if the system (1) is uniformly exponentially trichotomic then it is nonuniformly exponentially trichotomic. But the converse statement is not necessarily valid. Song et al. had ordered a counter example (in [9]) to illustrate the converse statement.

\section{Polynomial Trichotomy Concepts}

Definition 7. The linear discrete-time system (1) is said to be uniformly polynomially trichotomic if there exist three projection families $\left\{P_{i}\right\}_{i \in\{1,2,3\}}$ compatible with the system (1), constants $N \geq 1$, and $v_{1}, v_{2}, v_{3}$, and $v_{4}$ with the property $v_{1} \leq v_{2}<-1,1<v_{3} \leq v_{4}$ such that

$$
\begin{aligned}
& \left(\mathrm{upt}_{1}\right)\left\|U(m, n) P_{1}(n) x\right\| \leq N((m+1) /(n+1))^{v_{1}}\left\|P_{1}(n) x\right\|, \\
& \left(\mathrm{upt}_{2}\right)\left\|P_{2}(n) x\right\| \leq N((m+1) /(n+1))^{-v_{4}}\left\|U(m, n) P_{2}(n) x\right\|, \\
& \left(\mathrm{upt}_{3}\right)\left\|U(m, n) P_{3}(n) x\right\| \leq N((m+1) /(n+1))^{v_{3}}\left\|P_{3}(n) x\right\|, \\
& \left(\mathrm{upt}_{4}\right)\left\|P_{3}(n) x\right\| \leq N((m+1) /(n+1))^{-v_{2}}\left\|U(m, n) P_{3}(n) x\right\|,
\end{aligned}
$$

for all $(m, n, x) \in \Delta \times X$. 
Ramneanţu had shown an example for u.p.t in [5].

Definition 8. The linear discrete-time system (1) is said to be nonuniformly polynomially trichotomic if there exist three projection families $\left\{P_{i}\right\}_{i \in\{1,2,3\}}$ compatible with the system (1), a nondecreasing sequence of real numbers $N: \mathbb{N} \rightarrow \mathbb{R}_{+}^{*}$, and constants $v_{1}, v_{2}, v_{3}$, and $v_{4}$ with the property $v_{1} \leq v_{2}<$ $-1,1<v_{3} \leq v_{4}$ such that

(a) $\left\|U(m, n) P_{1}(n) x\right\| \leq N(n)((m+1) /(n+1))^{v_{1}}\left\|P_{1}(n) x\right\|$,

(b) $\left\|P_{2}(n) x\right\| \leq N(m)((m+1) /(n+1))^{-v_{4}} \| U(m$, n) $P_{2}(n) x \|$,

(c) $\left\|U(m, n) P_{3}(n) x\right\| \leq N(n)((m+1) /(n+1))^{v_{3}}\left\|P_{3}(n) x\right\|$,

(d) $\left\|P_{3}(n) x\right\| \leq N(m)((m+1) /(n+1))^{-v_{2}} \| U(m$, n) $P_{3}(n) x \|$,

for all $(m, n, x) \in \Delta \times X$.

Remark 9. (I) For $P_{3}=0$ in Definition 8 we obtain the property of nonuniform polynomial dichotomy.

(II) For $P_{2}=P_{3}=0$, the property of nonuniform polynomial stability is obtained. It follows that a nonuniformly polynomial stable linear discrete-time system is nonuniformly polynomially dichotomic and, further, nonuniformly polynomial trichotomic.

(III) For $P_{1}=P_{3}=0$, we obtain the property of nonuniform polynomial expansivity. Also it is easy to see that the property of nonuniform polynomial expansivity implies the nonuniform polynomial dichotomy and, further, the nonuniform polynomial trichotomy.

Remark 10. The linear discrete-time system (1) is nonuniformly polynomially trichotomic if and only if there exist a nondecreasing sequence of real numbers $N: \mathbb{N} \rightarrow \mathbb{R}_{+}^{*}$, constants $v_{1}, v_{2}, v_{3}$, and $v_{4}$ with the property $v_{1} \leq v_{2}<$ $-1,1<v_{3} \leq v_{4}$, and three projection families $\left\{P_{i}\right\}_{i \in\{1,2,3\}}$ compatible with the system (1) such that

$$
\begin{aligned}
&\left(\mathrm{npt}_{1}^{\prime}\right)\left\|U(m, n) P_{1}(n) x\right\| \leq N(p)((m+1) /(p+1))^{v_{1}} \\
&\left\|U(p, n) P_{1}(n) x\right\|, \\
&\left(\mathrm{npt}_{2}^{\prime}\right)\left\|U(p, n) P_{2}(n) x\right\| \leq N(m)((m+1) /(p+1))^{-v_{4}} \\
&\left\|U(m, n) P_{2}(n) x\right\|, \\
&\left(\mathrm{npt}_{3}^{\prime}\right)\left\|U(m, n) P_{3}(n) x\right\| \leq N(p)((m+1) /(p+1))^{v_{3}} \\
&\left\|U(p, n) P_{3}(n) x\right\|, \\
&\left(\mathrm{npt}_{4}^{\prime}\right)\left\|U(p, n) P_{3}(n) x\right\| \leq N(m)((m+1) /(p+1))^{-v_{2}} \\
&\left\|U(m, n) P_{3}(n) x\right\|,
\end{aligned}
$$

for all $(m, p, n, x) \in T \times X$.

Remark 11. It is obvious that if the system (1) is uniformly polynomially trichotomic then it is nonuniformly polynomially trichotomic. But the converse statement is not necessarily valid. This fact is illustrated by the following example.
Example 12. Let $X=\mathbb{R}^{3}, P_{1}(n) x=\left(x_{1}, 0,0\right), P_{2}(n) x=$ $\left(0, x_{2}, 0\right)$, and $P_{3}(n) x=\left(0,0, x_{3}\right)$. Considering $v:[1, \infty) \rightarrow$ $[1, \infty), k \in N$

$$
v(n)= \begin{cases}e^{m^{2}}, & m=3 k \\ e^{4}, & m=3 k+1 \\ 1, & m=3 k+2 .\end{cases}
$$

We consider the application

$$
\begin{aligned}
A(n)\left(x_{1}, x_{2}, x_{3}\right)= & \left(\frac{(n+1)^{2} v(n)}{(n+2)^{2} v(n+1)} x_{1},\right. \\
& \left.\frac{(n+2)^{2} v(n+1)}{(n+1)^{2} v(n)} x_{2}, \frac{(n+1)^{2} v(n)}{(n+2)^{2} v(n+1)} x_{3}\right) .
\end{aligned}
$$

We have the property

$$
\begin{aligned}
& \left(\frac{m+1}{n+1}\right)^{2}\left\|U(m, n) P_{1}(n) x\right\| \\
& \quad=\frac{(m+1)^{2}(n+1)^{2} v(n)}{(n+1)^{2}(m+1)^{2} v(m)}\left\|P_{1}(n) x\right\| \\
& \quad \leq v(n)\left\|P_{1}(n) x\right\| \\
& \quad \leq N(n)\left\|P_{1}(n) x\right\|, \\
& N(m)\left\|U(m, n) P_{2}(n) x\right\| \\
& \quad=N(m) \frac{(m+1)^{2} v(m)}{(n+1)^{2} v(n)}\left\|P_{2}(n) x\right\| \\
& \quad \geq\left(\frac{m+1}{n+1}\right)^{2}\left\|P_{2}(n) x\right\|, \\
& \left(\frac{n+1}{m+1}\right)^{2}\left\|U(m, n) P_{3}(n) x\right\| \\
& \quad=\frac{(n+1)^{2}(n+1)^{2} v(n)}{(m+1)^{2}(m+1)^{2} v(m)}\left\|P_{3}(n) x\right\| \\
& \quad \leq v(n)\left\|P_{3}(n) x\right\| \leq N(n)\left\|P_{3}(n) x\right\|, \\
& N(m)\left\|U(m, n) P_{3}(n) x\right\| \\
& \quad \geq\left(\frac{n+1}{n+m}\right)^{2}\left\|P_{3}(n) x\right\|, \\
& (m) \frac{(n+1)^{2} v(n)}{(m+1)^{2} v(m)}\left\|P_{3}(n) x\right\|
\end{aligned}
$$

for all $(m, n, x) \in \Delta \times X, N(n)=e^{n^{2}}$, and $-v_{1}=-v_{2}=v_{3}=$ $v_{4}=2$. Then system (1) is n.p.t.

If we suppose that system (1) is u.p.t, then there are constants $N \geq 1$ and $\alpha>1$, such that

$$
\left(\frac{m+1}{n+1}\right)^{\alpha} \frac{(n+1)^{2} v(n)}{(m+1)^{2} v(m)} \leq N, \quad \text { for }(m, n) \in \Delta .
$$


Then for $n=k$ and $m=3 k+1$ we obtain

$$
\left(1+\frac{1}{3 k+1}\right)^{\alpha}\left(1-\frac{1}{3 k+2}\right)^{2} \leq N \frac{e^{4}}{e^{k^{2}}}
$$

which for $k \rightarrow \infty$ gives a contradiction and hence system (1) is not u.p.t.

\section{The Main Results}

Theorem 13. The linear discrete-time system (1) is nonuniformly polynomially trichotomic if and only if there exist a function $F \in \mathscr{F}$ and three projection families $\left\{P_{i}\right\}_{i \in\{1,2,3\}}$ compatible with the system (1) such that following relations hold:

(i) there exist a constant $d_{1}>0$ and a sequence of positive real numbers $\left\{\xi_{n}\right\}_{n \in \mathbb{N}}$ such that

$$
\sum_{k=n}^{\infty} F\left(\left(\frac{k+1}{n+1}\right)^{d_{1}}\left\|U(k, n) P_{1}(n) x\right\|\right) \leq F\left(\xi_{n}\left\|P_{1}(n) x\right\|\right)
$$

(ii) there exist a constant $d_{2}>0$ and a sequence of positive real numbers $\left\{\eta_{n}\right\}_{n \in \mathbb{N}}$ such that

$$
\begin{aligned}
& \sum_{k=n}^{m} F\left(\left(\frac{k+1}{n+1}\right)^{-d_{2}}\left\|U(k, n) P_{2}(n) x\right\|\right) \\
& \quad \leq F\left(\eta_{m}\left(\frac{m+1}{n+1}\right)^{-d_{2}}\left\|U(m, n) P_{2}(n) x\right\|\right) ;
\end{aligned}
$$

(iii) there exist a constant $d_{3}>0$ and a sequence of positive real numbers $\left\{\alpha_{n}\right\}_{n \in \mathbb{N}}$ such that

$$
\sum_{k=n}^{\infty} F\left(\left(\frac{k+1}{n+1}\right)^{-d_{3}}\left\|U(k, n) P_{3}(n) x\right\|\right) \leq F\left(\alpha_{n}\left\|P_{3}(n) x\right\|\right)
$$

(iv) there exist a constant $d_{4}>0$ and a sequence of positive real numbers $\left\{\beta_{n}\right\}_{n \in \mathbb{N}}$ such that

$$
\begin{aligned}
& \sum_{k=n}^{m} F\left(\left(\frac{k+1}{n+1}\right)^{d_{4}}\left\|U(k, n) P_{3}(n) x\right\|\right) \\
& \quad \leq F\left(\beta_{m}\left(\frac{m+1}{n+1}\right)^{d_{4}}\left\|U(m, n) P_{3}(n) x\right\|\right),
\end{aligned}
$$

for all $(m, p, n, x) \in T \times X$.

Proof. (a) $\Leftrightarrow$ (i) Necessity. We consider $F(t)=t, t \geq$ 0 . As system (1) is nonuniformly polynomially trichotomic, Definition 8 assures the existence of constant $v_{1}<0$, a sequence of real numbers $N: \mathbb{N} \rightarrow \mathbb{R}_{+}^{*}$, and a projection family $P_{1}$ such that (a) holds. We obtain for $d_{1}=\left(-v_{1}-1\right) / 2>$ 0 and according to (a)

$$
\begin{aligned}
& \sum_{k=n}^{\infty}\left(\frac{k+1}{n+1}\right)^{d_{1}}\left\|U(k, n) P_{1}(n) x\right\| \\
& \leq \sum_{k=n}^{\infty} N(n)\left(\frac{k+1}{n+1}\right)^{\left(v_{1}-1\right) / 2}\left\|P_{1}(n) x\right\| \\
&=N(n)(n+1)^{\left(1-v_{1}\right) / 2}\left\|P_{1}(n) x\right\| \sum_{k=n}^{\infty}(k+1)^{\left(v_{1}-1\right) / 2} \\
&=N(n)(n+1)^{\left(1-v_{1}\right) / 2}\left\|P_{1}(n) x\right\| \\
& \quad \times\left[(n+1)^{\left(v_{1}-1\right) / 2}+\sum_{k=n+1}^{\infty}(k+1)^{\left(v_{1}-1\right) / 2}\right] \\
& \leq N(n)(n+1)^{\left(1-v_{1}\right) / 2}\left\|P_{1}(n) x\right\| \\
& \times\left[(n+1)^{\left(v_{1}-1\right) / 2}-\frac{2}{v_{1}+1}(n+1)^{\left(v_{1}+1\right) / 2}\right] \\
&= N(n)\left\|P_{1}(n) x\right\|\left(1-\frac{2(n+1)}{v_{1}+1}\right) \\
&= \xi_{n}\left\|P_{1}(n) x\right\| .
\end{aligned}
$$

Sufficiency. According to the hypothesis, if we consider for for all $k=m>n$ and $d_{1} \geq 1$ then

$$
F\left(\left(\frac{m+1}{n+1}\right)^{d_{1}}\left\|U(m, n) P_{1}(n) x\right\|\right) \leq F\left(\xi_{n}\left\|P_{1}(n) x\right\|\right) .
$$

Similarly the other equivalences can also be proved; that is, (b) $\Leftrightarrow$ (ii), (c) $\Leftrightarrow$ (iii), and (d) $\Leftrightarrow$ (iv).

Remark 14. It is obvious that if the nonuniform trichotomy system (1) is nonuniformly exponentially trichotomic, then it is nonuniformly polynomially trichotomic, which means polynomial sense is more wide than the exponent's.

Definition 15. Two applications $L_{1}, L_{2}: \Delta \times X \rightarrow \mathbb{R}_{+}$ are called to be nonuniform polynomial Lyapunov functions for system (1) if there are a nondecreasing sequence of real number $K: \mathbb{R}_{+} \rightarrow[1, \infty), F \in \mathscr{F}$, and constants $l_{1}, l_{2}>0$ such that

(1) $L_{1}\left(m, r, P_{1}(r) x\right)+\sum_{k=n}^{m-1} F\left(((k+1) /(n+1))^{l_{1}} \| U(k\right.$, $\left.r) P_{1}(r) x \|\right) \leq L_{1}\left(n, r, P_{1}(r) x\right)$;

(2) $L_{2}\left(n, r, P_{2}(r) x\right)+\sum_{k=n+1}^{m} F\left(((k+1) /(m+1))^{-l_{2}} \| U(k\right.$, $\left.r) P_{1}(r) x \|\right) \leq L_{2}\left(m, r, P_{2}(r) x\right)$

(3) $L_{1}\left(m, r, P_{3}(r) x\right) \leq L_{1}\left(n, r, P_{3}(r) x\right)$;

(4) $L_{2}\left(n, r, P_{3}(r) x\right) \leq L_{2}\left(m, r, P_{3}(r) x\right)$;

(5) $L_{1}(m, r, x) \leq K(r)\left\|P_{1}(r) x\right\|+K(m)\left\|U_{3}(m, r) x\right\|$;

(6) $L_{2}(m, r, x) \leq K(m)\left\|U_{2}(m, r) x\right\|+K(r)\left\|P_{3}(r) x\right\|$;

(7) $L_{1}\left(m, r, P_{2}(r) x\right)=L_{2}\left(m, r, P_{1}(r) x\right)=0$,

for all $(m, n, r, x) \in T \times X$. 
Theorem 16. System (1) is nonuniformly polynomially trichotomic if and only if there exist nonuniform polynomial Lyapunov functions for it.

Proof. Necessity. Let $d_{1}, d_{2}, d_{3}$, and $d_{4}$ be given by Theorem 13. We consider the applications $L_{1}, L_{2}: \Delta \times X \rightarrow$ $\mathbb{R}_{+}$:

$$
\begin{aligned}
L_{1}(m, r, x)= & \sum_{k=m}^{\infty} F\left(\left(\frac{k+1}{m+1}\right)^{d_{1}}\left\|U(k, r) P_{1}(r) x\right\|\right) \\
& +\sum_{k=r}^{m} F\left(\left(\frac{k+1}{m+1}\right)^{d_{4}}\left\|U(k, r) P_{3}(r) x\right\|\right), \\
L_{2}(m, r, x)= & \sum_{k=r}^{m} F\left(\left(\frac{k+1}{m+1}\right)^{-d_{2}}\left\|U(k, r) P_{2}(r) x\right\|\right) \\
& +\sum_{k=m}^{\infty} F\left(\left(\frac{k+1}{m+1}\right)^{-d_{3}}\left\|U(k, r) P_{3}(r) x\right\|\right) .
\end{aligned}
$$

Then, for $m \geq n \geq r \geq 0$ and $x \in X$, we have

$$
\begin{aligned}
& L_{1}\left(m, r, P_{1}(r) x\right)+\sum_{k=n}^{m-1} F\left(\left(\frac{k+1}{n+1}\right)^{d_{1}}\left\|U(k, r) P_{1}(r) x\right\|\right) \\
&=\sum_{k=m}^{\infty} F\left(\left(\frac{k+1}{m+1}\right)^{d_{1}}\left\|U(k, r) P_{1}(r) x\right\|\right) \\
& \quad+\sum_{k=n}^{m-1} F\left(\left(\frac{k+1}{n+1}\right)^{d_{1}}\left\|U(k, r) P_{1}(r) x\right\|\right) \\
& \leq \sum_{k=n}^{\infty} F\left(\left(\frac{k+1}{n+1}\right)^{d_{1}}\left\|U(k, r) P_{1}(r) x\right\|\right) \\
&=L_{1}\left(n, r, P_{1}(r) x\right), \\
& L_{2}\left(n, r, P_{2}(r) x\right)+\sum_{k=n+1}^{m} F\left(\left(\frac{k+1}{m+1}\right)^{-d_{2}}\left\|U(k, r) P_{2}(r) x\right\|\right) \\
&=\sum_{k=r}^{n} F\left(\left(\frac{k+1}{n+1}\right)^{-d_{2}}\left\|U(k, r) P_{2}(r) x\right\|\right) \\
& \quad+\sum_{k=n}^{m-1} F\left(\left(\frac{k+1}{m+1}\right)^{-d_{2}}\left\|U(k, r) P_{2}(r) x\right\|\right) \\
& \leq \sum_{k=m}^{\infty} F\left(\left(\frac{k+1}{m+1}\right)^{-d_{2}}\left\|U(k, r) P_{2}(r) x\right\|\right) \\
&= L_{2}\left(m, r, P_{2}(r) x\right), \\
& L_{1}\left(m, r, P_{3}(r) x\right) \leq \sum_{k=r}^{n} F\left(\left(\frac{k+1}{n+1}\right)^{d_{4}}\left\|U(k, r) P_{3}(r) x\right\|\right) \\
& L_{1}\left(n, r, P_{3}(r) x\right),
\end{aligned}
$$

$$
\begin{aligned}
L_{2}\left(n, r, P_{3}(r) x\right) & \leq \sum_{k=m}^{\infty} F\left(\left(\frac{k+1}{m+1}\right)^{-d_{3}}\left\|U(k, r) P_{3}(r) x\right\|\right) \\
& =L_{2}\left(m, r, P_{3}(r) x\right) .
\end{aligned}
$$

By Theorem 13, for $F(t)=t$, we simply have

$$
\begin{aligned}
L_{1}(m, r, x) & \leq \xi_{r}\left\|P_{1}(r) x\right\|+\alpha_{r}\left\|P_{3}(r) x\right\| \\
& \leq K(r)\left[\left\|P_{1}(r) x\right\|+\left\|P_{3}(r) x\right\|\right], \\
L_{2}(m, r, x) & \leq \eta_{r}\left\|U_{2}(m, r) x\right\|+\beta_{r}\left\|U_{3}(m, r) x\right\| \\
& \leq K(m)\left[\left\|U_{2}(m, r) x\right\|+\left\|U_{3}(m, r) x\right\|\right] .
\end{aligned}
$$

We obtain that there are a nondecreasing sequence of real number $K: \mathbb{R}_{+} \rightarrow[1, \infty), F \in \mathscr{F}$, and constants $l_{1}, l_{2}>0$ such that the conditions of Definition 15 hold.

Sufficiency. Let $(m, n, r, x) \in T \times X$. If $L, L_{2}: \Delta \times X \rightarrow \mathbb{R}_{+}$ are nonuniform polynomial Lyapunov functions for system (1) then there exist $K: \mathbb{R}_{+} \rightarrow[1, \infty)$ and $L_{1}, L_{2}, L_{3}$, and $L_{4}$ such that the conditions of Definition 15 hold. We have that

$$
\begin{aligned}
& \sum_{k=n}^{\infty} F\left(\left(\frac{k+1}{n+1}\right)^{d_{1}}\left\|U(k, n) P_{1}(n) x\right\|\right) \\
&+\sum_{k=n}^{m} F\left(\left(\frac{k+1}{m+1}\right)^{d_{4}}\left\|U(k, n) P_{3}(n) x\right\|\right) \\
& \leq L_{1}\left(n, n, P_{1}(n) x\right)+L_{1}\left(m, n, P_{3}(n) x\right) \\
& \leq K(n)\left\|P_{1}(n) x\right\|+K(m)\left\|U_{3}(m, n) x\right\|, \\
& \sum_{k=n}^{m} F\left(\left(\frac{k+1}{m+1}\right)^{-d_{2}}\left\|U(k, n) P_{2}(n) x\right\|\right) \\
& \quad+\sum_{k=n}^{\infty} F\left(\left(\frac{k+1}{n+1}\right)^{-d_{3}}\left\|U(k, n) P_{3}(n) x\right\|\right) \\
& \leq L_{2}\left(m, n, P_{2}(n) x\right)+L_{2}\left(n, n, P_{3}(n) x\right) \\
& \leq K(m)\left\|U_{2}(m, n) x\right\|+K(n)\left\|P_{3}(n) x\right\|
\end{aligned}
$$

for all $(m, n, r, x) \in T \times X$. Using Theorem 13 we obtain that system (1) is n.p.t.

\section{Conflict of Interests}

The authors declare that there is no conflict of interests regarding the publication of this paper.

\section{Acknowledgments}

The authors would like to thank the referee for helpful suggestions and comments. This work was supported by the Fundamental Research Funds for the Central Universities (no. 2013XK03). 


\section{References}

[1] L. Barreira and C. Valls, "Polynomial growth rates," Nonlinear Analysis: Theory, Methods and Applications, vol. 71, no. 11, pp. 5208-5219, 2009.

[2] A. J. G. Bento and C. Silva, "Stable manifolds for nonuniform polynomial dichotomies," Journal of Functional Analysis, vol. 257, no. 1, pp. 122-148, 2009.

[3] M. L. Ramneanţu, T. Ceausu, and M. Megan, "On nonuniform polynomial dichotomy of evolution operators in Banach Spaces," International Journal of Pure and Applied Mathematics, vol. 75, no. 3, pp. 305-518, 2012.

[4] M. Megan and C. Stoica, "On uniform exponential trichotomy of evolution operators in Banach spaces," Integral Equations and Operator Theory, vol. 64, no. 4, pp. 499-506, 2008.

[5] M. L. Ramneanţu, "Uniform polynomial trichotomy of evolution operators in Banach spaces," Journal of Advanced Mathematical Studies, vol. 5, no. 2, pp. 101-106, 2012.

[6] O. Perron, "Die Stabilitatsfrage bei Differentialgleichungen," Mathematische Zeitschrift, vol. 32, no. 1, pp. 703-728, 1930.

[7] R. J. Sacker and G. R. Sell, "Existence of dichotomies and invariant splittings for linear differential systems, III," Journal of Differential Equations, vol. 22, no. 2, pp. 497-522, 1976.

[8] B. Sasu and A. L. Sasu, "Nonlinear criteria for the existence of the exponential trichotomy in infinite dimensional spaces," Nonlinear Analysis: Theory, Methods and Applications, vol. 74, no. 15, pp. 5097-5110, 2011.

[9] X. Song, T. Yue, and D. Li, "Nonuniform exponential trichotomy for linear discrete-time systems in Banach spaces," Journal of Function Spaces and Applications, vol. 2013, Article ID 645250, 6 pages, 2013.

[10] L. Barreira and C. Valls, "Lyapunov functions for trichotomies with growth rates," Journal of Differential Equations, vol. 248, no. 1, pp. 151-183, 2010.

[11] S. Elaydi and O. Hájek, "Exponential trichotomy of differential systems," Journal of Mathematical Analysis and Applications, vol. 129, no. 2, pp. 362-374, 1988.

[12] S. Elaydi and O. Hájek, "Exponential dichotomy and trichotomy of nonlinear differential equations," Differential and Integral Equations, vol. 3, no. 6, pp. 1201-1224, 1990.

[13] S. Elaydi and K. Janglajew, "Dichotomy and trichotomy of difference equations," Journal of Difference Equations and Applications, vol. 3, no. 5-6, pp. 417-448, 1998.

[14] A. I. Alonso, J. Hong, and R. Obaya, "Exponential dichotomy and trichotomy for difference equations," Computers \& Mathematics with Applications, vol. 38, no. 1, pp. 41-49, 1999. 


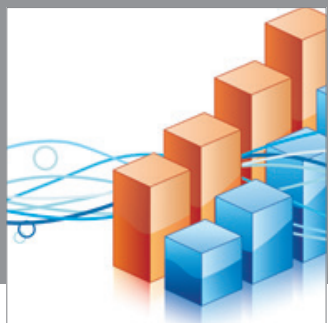

Advances in

Operations Research

mansans

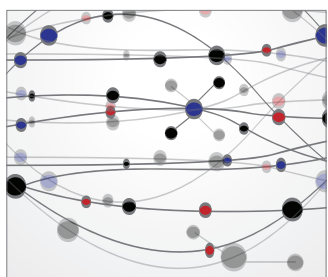

The Scientific World Journal
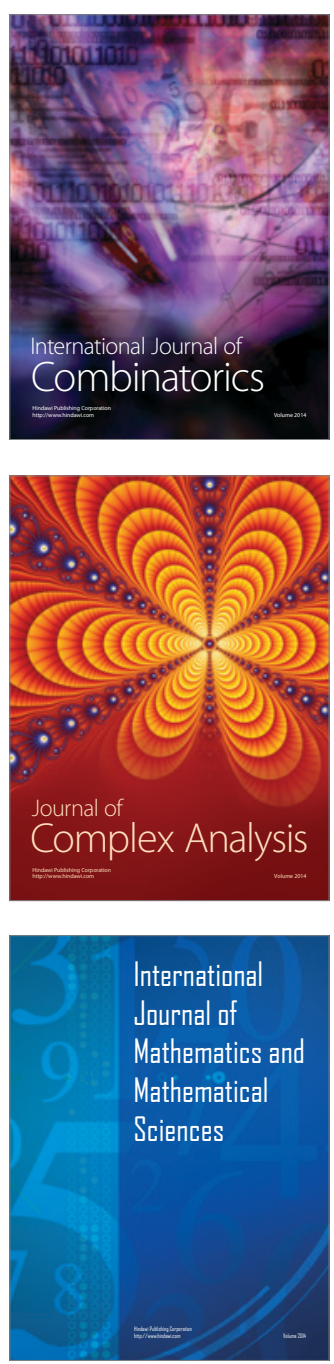
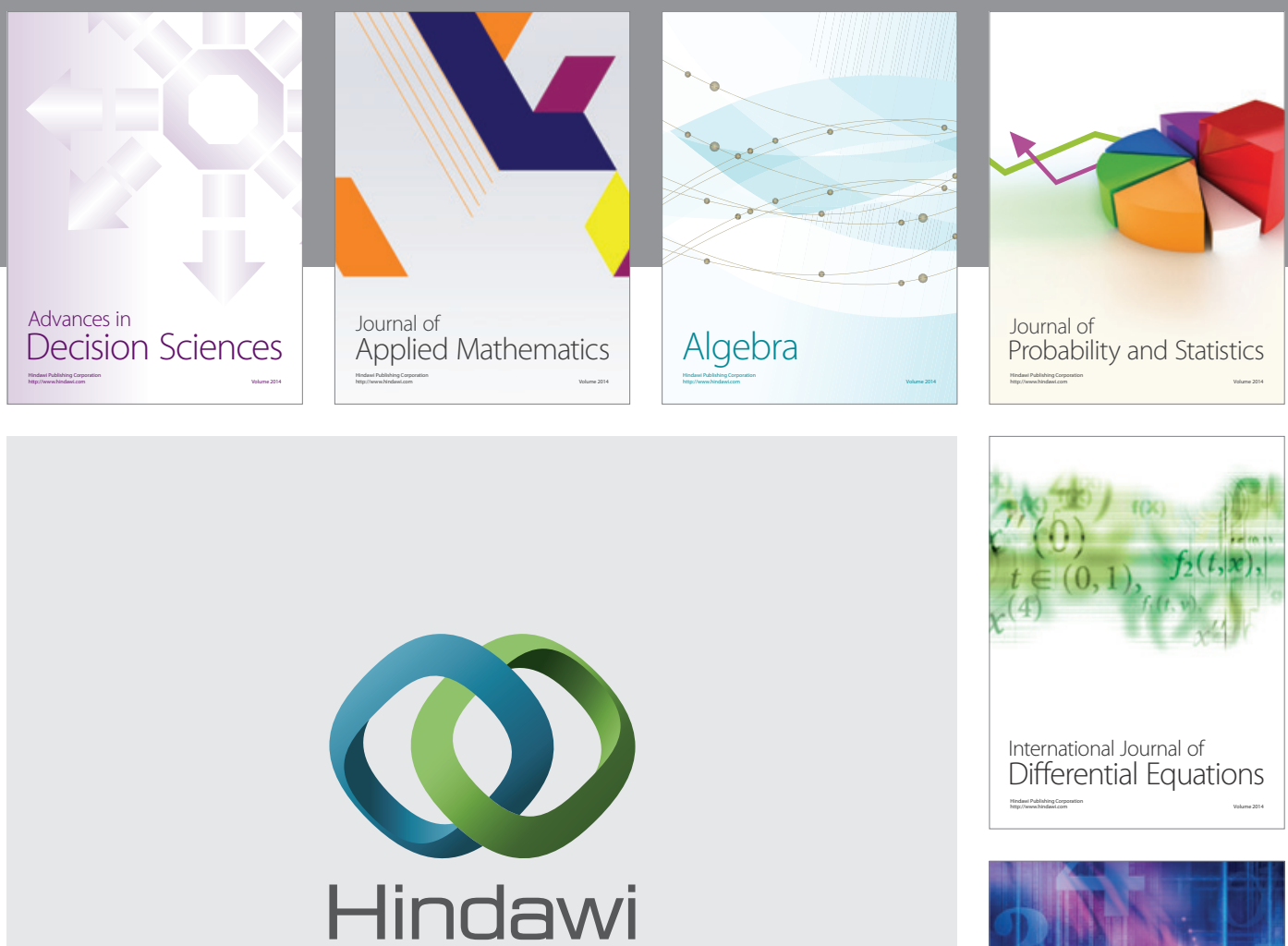

Submit your manuscripts at http://www.hindawi.com
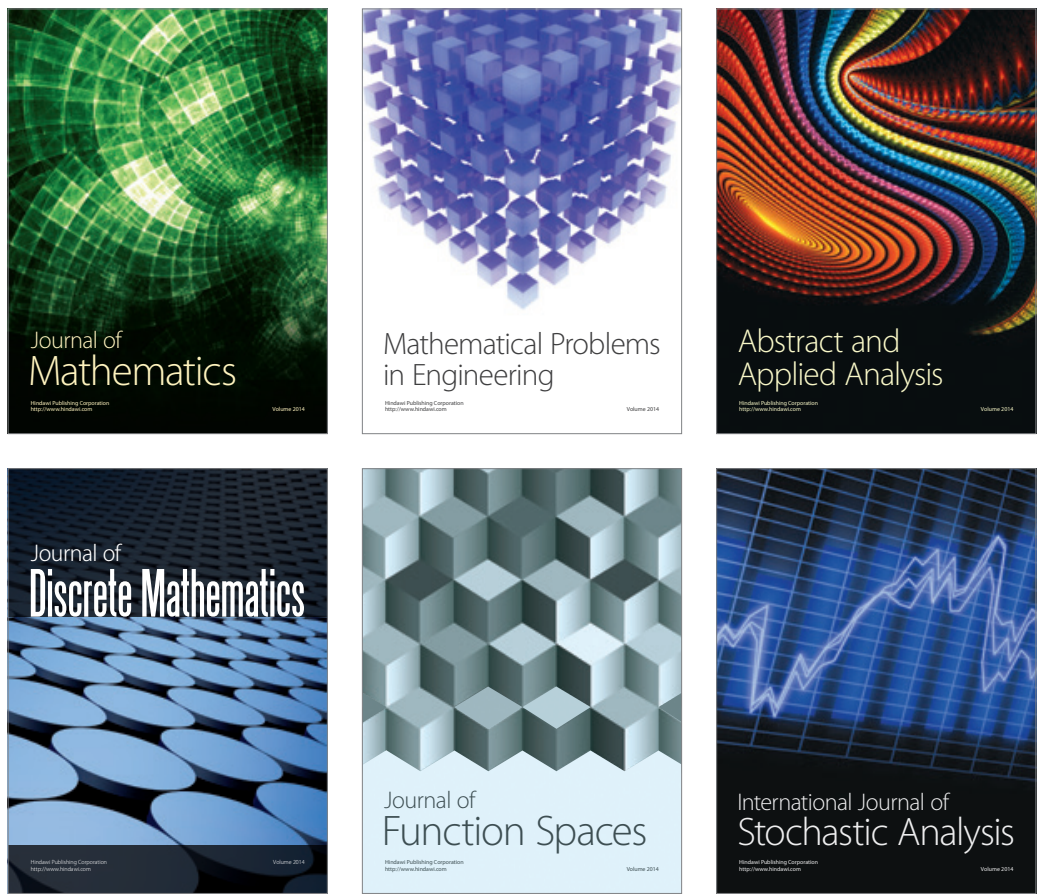

Journal of

Function Spaces

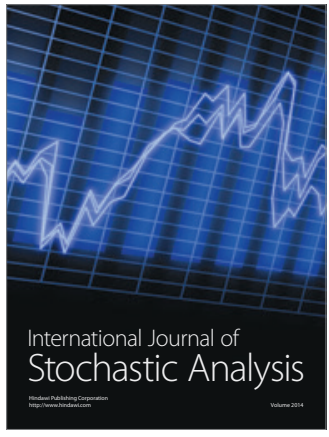

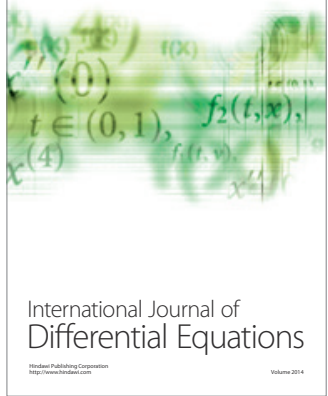
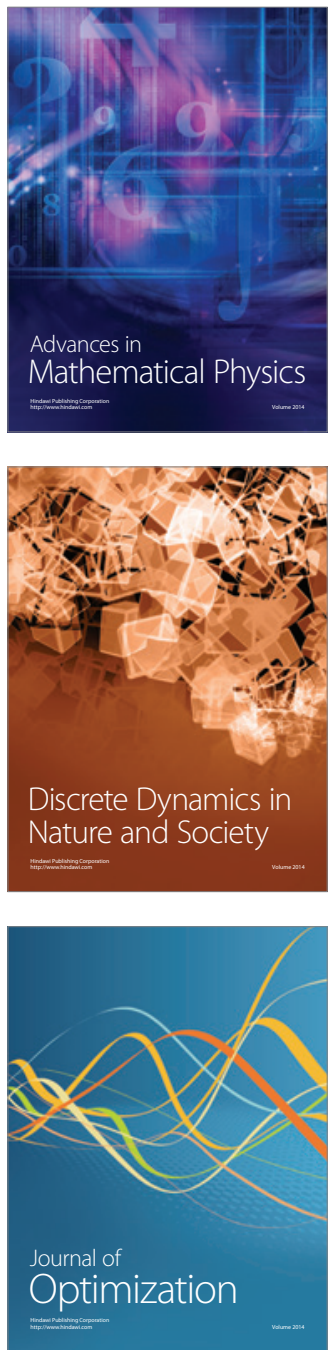